\title{
The domain of attraction of the $\alpha$-sun operator for type II and type III distributions
}

\author{
GERARD HOOGHIEMSTRA ${ }^{1 *}$ and PRISCILLA E. GREENWOOD ${ }^{2}$ \\ ${ }^{I}$ Delft University of Technology, Faculty of Technical Mathematics and Computer Science, \\ Mekelweg 4, 2628 CD Delft, The Netherlands \\ ${ }^{2}$ University of British Columbia, Mathematical Institute, 121-1984 Mathematics Road, \\ Vancouver, British Columbia, Canada V6T 1 Y4
}

Let $\left(Y_{n}\right)$ be a sequence of independent random variables with common distribution $F$ and define the iteration: $X_{0}=x_{0}, X_{n}:=X_{n-1} \vee\left(\alpha X_{n-1}+Y_{n}\right), \alpha \in[0,1)$. We denote by $\mathscr{D}\left(\Phi_{\gamma}\right)$ the domain of maximal attraction of $\Phi_{\gamma}$, the extreme value distribution of the first type. Greenwood and Hooghiemstra showed in 1991 that for $F \in \mathscr{D}\left(\Phi_{\gamma}\right)$ there exist norming constants $a_{n}>0$ and $b_{n} \in \mathbf{R}$ such that $a_{n}^{-1}\left\{X_{n}-b_{n} /(1-\alpha)\right\}$ has a non-degenerate (distributional) limit. In this paper we show that the same is true for $F \in \mathscr{D}\left(\Psi_{\gamma}\right) \cup \mathscr{D}(\Lambda)$, the type II and type III domains. The method of proof is entirely different from the method in the aforementioned paper. After a proof of tightness of the involved sequences we apply (modify) a result of Donnelly, concerning weak convergence of Markov chains with an entrance boundary.

Keywords: extremal limits; self-similar Markov processes; weak convergence

\section{Introduction}

Let $\left(Y_{n}\right)_{n \geqslant 1}$ be a sequence of independent random variables with common distribution function $F$ and define the iteration

$$
X_{0}=x_{0}, X_{n}:=X_{n-1} \vee\left(\alpha X_{n-1}+Y_{n}\right), n \geqslant 1, \alpha \in[0,1) .
$$

We denote by $\mathscr{D}(G)$ the domain of maximal attraction of the distribution $G$, where $G$ is one of the extreme value distributions. For $F \in \mathscr{D}(G)$ and $a_{n}>0, b_{n} \in \mathbf{R}$ such that $F^{n}\left(a_{n} x+b_{n}\right) \rightarrow G(x)$, for all $x$, we define, for $n \geqslant 1$,

$$
Y_{n, j}:=\frac{Y_{j}-b_{n}}{a_{n}}, \quad j=1,2, \ldots
$$

For $\alpha \in[0,1)$ and $x_{0} \in \mathbf{R}$, the random element $X_{n}(\cdot) \in D[0, \infty)$ (the space of cadlag functions, equipped with the Skorohod topology) is defined by

\footnotetext{
* To whom correspondence should be addressed
} 


$$
X_{n}(t):= \begin{cases}a_{n}^{-1}\left(x_{0}-\frac{b_{n}}{1-\alpha}\right), & 0 \leqslant t<n^{-1}, \\ X_{n}\left(\frac{j-1}{n}\right) \vee\left\{\alpha X_{n}\left(\frac{j-1}{n}\right)+Y_{n, j}\right\}, & \frac{j}{n} \leqslant t<\frac{j+1}{n}, j=1,2, \ldots\end{cases}
$$

Observe that the relation between the sequence of random variables $X_{n}$ given by (1) and the sequence of processes $X_{n}(\cdot)$ is

$$
X_{n}\left(\frac{j}{n}\right)=a_{n}^{-1}\left(X_{j}-\frac{b_{n}}{1-\alpha}\right) .
$$

The motivation for studying recursive sequences such as (1) comes from a stochastic solar energy model (cf. Haslett 1980). Note that for $\alpha=0$ the sequence $X_{n}$ is the sequence of partial maxima:

$$
X_{n}=x_{0} \vee Y_{1} \vee \cdots \vee Y_{n}
$$

whereas for $\alpha=1$ (this value is not included in the definition (1)) we obtain

$$
X_{n}=x_{0}+\left[Y_{1}\right]^{+}+\cdots+\left[Y_{n}\right]^{+} \quad\left([x]^{+}=x \vee 0, x \in \mathbf{R}\right) .
$$

Hence the sequence $X_{n}$ defined by (1) is between maxima and sums of independent random variables, and from that viewpoint of theoretical interest.

Greenwood and Hooghiemstra (1991) showed that for $F \in \mathscr{D}\left(\Phi_{\gamma}\right)$, where

$$
\Phi_{\gamma}(x):=\exp \left(-x^{-\gamma}\right) 1_{[0, \infty)}(x)
$$

the process $X_{n}(\cdot)$ converges weakly in $D[0, \infty)$ to a self-similar Markov process $Z(\cdot)$. Furthermore the distribution of $Z(1)$ admits a density $h_{\alpha}$ on $(0, \infty)$, given as the unique density solution of the equation

$$
h_{\alpha}(x)=\frac{\gamma}{x} \int_{0}^{x}(x-\alpha u)^{-\gamma} h_{\alpha}(u) \mathrm{d} u, \quad x>0 .
$$

In this case $X_{n}(0)=a_{n}^{-1}\left\{x_{0}-b_{n} /(1-\alpha)\right\} \rightarrow 0$, and the proof proceeds by showing that the functional induced by (2) on the point process $\sum \delta_{\left(j / n, Y_{n, j}\right)}$ is continuous.

In this paper we prove weak convergence of $X_{n}(\cdot)$ for $F \in \mathscr{D}\left(\Psi_{\gamma}\right) \cup \mathscr{D}(\Lambda)$, where

$$
\begin{aligned}
\Psi_{\gamma}(x) & :=\exp \left[-(-x)^{\gamma}\right] 1_{(-\infty, 0]}(x)+1_{(0, \infty)}(x), \\
\Lambda(x) & :=\exp \left(-\mathrm{e}^{-x}\right) .
\end{aligned}
$$

For $F \in \mathscr{D}\left(\Psi_{\gamma}\right) \cup \mathscr{D}(\Lambda)$ we have $X_{n}(0)=a_{n}^{-1}\left\{x_{0}-b_{n} /(1-\alpha)\right\} \rightarrow-\infty$. In these cases the method of proof is entirely different from that in the work of Greenwood and Hooghiemstra (1991). It is based on the weak convergence of Markov processes to a limiting Markov process with entrance boundary. The proof uses monotonicity of the relevant Markov process and tightness of the sequence $X_{n}(t)$ for fixed positive $t$. In Sections 2 and 3 we prove weak convergence, aside from the tightness of $X_{n}(t)$, which we postpone to Section 4 . 


\section{The convergence result for type II distributions}

Let $F \in \mathscr{D}\left(\Psi_{\gamma}\right)$; then $r:=\sup \{x: F(x)<1\}<\infty$, and $1-F\left(r-x^{-1}\right)=x^{-\gamma} L(x)$, with $L$ slowly varying at infinity. Set $b_{n} \equiv r$ and $a_{n}:=r-\inf \left\{y: 1-F(y) \leqslant n^{-1}\right\}$. The points $\left(j / n, Y_{n, j}\right), n \geqslant 1, j=1,2, \ldots$ are contained in $E:=(0, \infty) \times(-\infty, 0)$. To prepare for the formulation of the convergence result we first specify what will be the limiting Markov process. Denote by $N$ a Poisson point process on $E$ with intensity measure the product of Lebesgue measure $\mathrm{d} t$ and the measure $\mathrm{d} \mu$, where

$$
\mu(y, 0)=|y|^{\gamma}, y<0 .
$$

For $x<0$ we denote by $N_{x}$ the points of $N$ in the strip $(0, \infty) \times[x, 0)$. We order the points of $N_{x}$ according to the first coordinate and denote them by $\left(t_{1}, j_{1}\right),\left(t_{2}, j_{2}\right), \ldots$, where $0<t_{1}<t_{2}<\cdots$ and $j_{k} \in[x, 0)$. The continuous-time Markov process $Z_{x}(\cdot)$ with state space $[x, 0)$ is defined by

$$
Z_{x}(t):= \begin{cases}x, & 0 \leqslant t<t_{1}, \\ Z_{x}\left(t_{k-1}\right) \vee\left\{\alpha Z_{x}\left(t_{k-1}\right)+j_{k}\right\}, & t_{k} \leqslant t<t_{k+1} .\end{cases}
$$

We shall show that, for $x \rightarrow-\infty$, the process $Z_{x}(\cdot)$ converges almost surely to a process $Z(\cdot)$ with $Z(0)=-\infty$, almost surely, whereas, for any $t>0$, we have $-\infty<Z(t)<0$, almost surely, and where the conditional distribution of $(Z(s) \mid Z(t)=x)$ is given by the distribution of $Z_{x}(s-t), s>t$. This final statement is clear from the definition of $Z_{x}$. The process $Z(\cdot)$ will be the limit of $X_{n}(\cdot)$ on $D(0, \infty)$. Here is a proof of the statements concerning $Z(\cdot)$.

Since we have, for $x<y$ and each $t \geqslant 0$,

$$
Z_{x}(t) \leqslant Z_{y}(t) \leqslant 0,
$$

the almost sure convergence of $Z_{x}(t)$ to a value $Z(t)$, possibly $-\infty$, follows. As for each $x$ the process $Z_{x}(\cdot)$ is non-decreasing we obtain that $Z(\cdot)$ is non-decreasing and we hence conclude that $Z_{x}(\cdot)$ converges almost surely to a non-decreasing random function $Z(\cdot)$, as $x \rightarrow-\infty$. If we show that for arbitrary $t>0$ the collection II $:=\left\{Z_{x}(t), x<0\right\}$ is uniformly tight, then $-\infty<Z(t) \leqslant 0, t>0$. The tightness of II is a consequence of the three lemmas below, the first of which goes back to Rényi and is well known.

Lemma 1. Fix $x<0$. Let $\sigma_{j}, j=1,2, \ldots$ be the points of a Poisson process on $\mathbf{R}^{+}$with intensity $|x|^{\gamma}$. Independent of this Poisson process we define an independent, identically distributed sequence $\beta_{1}, \beta_{2}, \ldots$ with distribution

$$
P\left(\beta_{1} \leqslant y\right)=1-\left|\frac{y}{x}\right|^{\gamma}, \quad x \leqslant y \leqslant 0 .
$$

Then the point process $N_{x}^{\prime}:=\sum_{j} \delta_{\left(\sigma_{j}, \beta_{j}\right)}$ is equal in distribution to $N_{x}$.

Lemma 2. Let $\left(X_{n}\right)$ be defined by (1) with initial value $X_{1}=-1$, and with $\left(Y_{n}\right)$ an independent, identically distributed sequence with distribution

$$
F(y)=1-|y|^{\gamma}, \quad-1 \leqslant y \leqslant 0 .
$$


Then

$$
\sup _{n \geqslant 1} n^{1 / \gamma} \mathrm{E} X_{n} \geqslant A,
$$

where $A<0$ is given by $|A|^{\gamma}:=\{(1+\gamma) / \gamma\}(1-\alpha)^{-1-\gamma}$.

Remark 1. Note that $F$ given in (5) belongs to $\mathscr{D}\left(\Psi_{\gamma}\right)$ and that for this specific distribution the norming constants are given by $b_{n}=0$ and $a_{n}=n^{-1 / \gamma}$. The proof below is equal to the tightness proof of Theorem 3 in Section 4 for $F$ given in (5). Because of the smoothness of $F$ the proof of Lemma 2 is easier than that of Theorem 3.

Proof. The conditional expectation $\mathrm{E}\left(X_{n+1} \mid X_{n}\right)=X_{n}+\int_{(1-\alpha) X_{n}}^{0}\{1-F(y)\} \mathrm{d} y$; so by taking double expectations and using the Jensen inequality

$$
\mathrm{E} X_{n+1}=\mathrm{E} g\left(X_{n}\right) \geqslant g\left(\mathrm{E} X_{n}\right),
$$

where $g(u):=u+\{(1-\alpha)|u|\}^{1+\gamma} /(1+\gamma),-1 \leqslant u \leqslant 0$. Put $u_{n}:=\mathrm{E} X_{n}$ and $v_{n}:=A n^{-1 / \gamma}$. We shall prove by induction that $u_{n} \geqslant v_{n}$ for all $n \geqslant 1$. For $n=1, u_{1}=-1$ and $v_{1}=A<-1$. Assume that $u_{n} \geqslant v_{n}$ for some $n$. By (6) and the monotonicity of $g$,

$$
u_{n+1} \geqslant g\left(u_{n}\right) \geqslant g\left(v_{n}\right) \text {. }
$$

The inequality $g\left(v_{n}\right) \geqslant v_{n+1}$ follows because $n\left[1-\{n /(n+1)\}^{1 / \gamma}\right] \leqslant 1 / \gamma$, for all $n \geqslant 1$ and $\gamma>0$.

Lemma 3. For any $t>0$,

$$
\lim _{M \rightarrow \infty} \lim _{x \rightarrow-\infty} P\left(Z_{x}(t) \geqslant-M\right)=1 .
$$

Proof. By monotonicity it is sufficient to show (7) for a sequence $x_{n} \rightarrow-\infty$. Let

$$
\tau_{n}:=\inf \left\{s>0: \# \text { points of } N \text { contained in the set }(0, s] \times\left[-n^{1 / \gamma}, 0\right)=n\right\} .
$$

Observe from Lemma 1 that, for $x_{n}=-n^{1 / \gamma}$, there holds $Z_{x_{n}}\left(\tau_{n}\right) \stackrel{d}{=} n^{1 / \gamma} X_{n}$, if $X_{1}:=-1$ and $F$ given in (5). Because $N$ is a Poisson process with intensity $\mathrm{d} t \times \mathrm{d} \mu$ the random variable $\tau_{n}$ is the sum of $n$ independent and exponentially distributed random variables each with parameter $n$. It is straightforward that $\tau_{n} \rightarrow 1$, a.s. Hence it follows from Lemma 2 and the monotonicity of $Z_{x}(\cdot)$ that for each $t>1$ the statement (7) holds. The result for $0<t \leqslant 1$ is easily obtained by noting that for any subsequence $n_{k}$ we have, with $m_{k}=\left[n_{k} t\right]$,

$$
\lim _{k \rightarrow \infty} n_{k}^{1 / \gamma} X_{\left[n_{k} t\right]}=t^{-1 / \gamma} \lim _{k \rightarrow \infty} m_{k}^{1 / \gamma} X_{m_{k}} .
$$

We now formulate and prove our main result for $F \in \mathscr{D}\left(\Psi_{\gamma}\right)$.

Theorem 1. Let $F \in \mathscr{D}\left(\Psi_{\gamma}\right)$ and $x_{0}<r /(1-\alpha)$. On $D(0, \infty)$ we have

$$
X_{n}(\cdot) \stackrel{d}{\rightarrow} Z(\cdot)
$$

where $Z(\cdot)$ is the Markov process with entrance boundary introduced above. 
Proof. The coordinate projection $X_{n}(t)$ at time $t>0$ is uniformly tight as a consequence of Theorem 3 in Section 4, because

$$
\lim _{n \rightarrow \infty} \frac{a_{[n t]}}{a_{n}}=t^{-1 / \gamma}
$$

and

$$
X_{n}(t)=a_{n}^{-1}\left(X_{[n t]}-\frac{r}{1-\alpha}\right)=\frac{a_{[n t]}}{a_{n}} a_{[n t]}^{-1}\left(X_{[n t]}-\frac{r}{1-\alpha}\right) .
$$

Next we check that the sequence $X_{n}(\cdot)$ is tight in $D[a, b]$, the space of cadlag functions with $t \in[a, b]$ for each pair $a, b$ with $0<a<b<\infty$. Given that $X_{n}(a)=x \in[-M, 0]$, the process $X_{n}(t), t \geqslant a$, is non-decreasing and converges weakly to $Z_{x}(t-a), t \geqslant a$, because of convergence of the underlying point processes and continuity of the map $(x, y) \rightarrow x \vee(\alpha x+y)$. Hence, if $n_{k}$ is a subsequence for which $X_{n_{k}}(a)$ converges weakly on $\mathbf{R}$, then $X_{n_{k}}(\cdot)$ converges weakly on $D[a, b]$. Consequently the sequence $X_{n}$ is relatively compact on $D[a, b]$ (and hence tight by Prohorov's theorem).

Take a particular weakly convergent subsequence of $X_{n}(\cdot)$ and denote its limit by $\hat{Z}(\cdot) \in D(0, \infty)$ (for convenience we shall also index the subsequence by $n$ ). For $t>0$ we denote by $\mathscr{C}_{t}$ the set of continuity points of the distribution of $\hat{Z}(t)$. We shall show that the process $\hat{Z}(\cdot)$ satisfies the following.

(i) For each $M>0, \lim _{h \downarrow 0} P(\hat{Z}(h) \leqslant-M)=1$.

(ii) For $0<s<t, x \in \mathscr{C}_{s}$ and $y \in \mathscr{C}_{t}$,

$$
P(\hat{Z}(s) \leqslant x, \hat{Z}(t) \leqslant y)=\int_{-\infty}^{z} P(\hat{Z}(s) \in \mathrm{d} u) P\left(Z_{u}(t-s) \leqslant y\right) .
$$

(iii) The finite-dimensional distributions of $\hat{Z}(\cdot)$ coincide with those of $Z(\cdot)$.

From (iii) the theorem follows, because the finite-dimensional distributions form a determining class.

If $-M \in \mathscr{C}_{h}$, then

$$
\begin{aligned}
P(\hat{Z}(h) \leqslant-M) & =\lim _{n \rightarrow \infty} P\left(X_{n}(h) \leqslant-M\right) \\
& \geqslant \lim _{n \rightarrow \infty} P\left(\sup _{1 \leqslant j \leqslant[n h]} Y_{n, j} \leqslant-M(1-\alpha)\right) \\
& =\exp \left\{-h M^{\gamma}(1-\alpha)^{\gamma}\right\} \rightarrow 1, h \downarrow 0 .
\end{aligned}
$$

This proves (i).

For $0<s<t, x \in \mathscr{C}_{s}$ and $y \in \mathscr{C}_{t}$,

$$
\begin{aligned}
P(\hat{Z}(s) \leqslant x, \hat{Z}(t) \leqslant y) & =\lim _{n \rightarrow \infty} P\left(X_{n}(s) \leqslant x, X_{n}(t) \leqslant y\right) \\
& =\lim _{n \rightarrow \infty} \int_{-\infty}^{x} P\left(X_{n}(s) \in \mathrm{d} u\right) P\left(X_{n}(t) \leqslant y \mid X_{n}(s)=u\right) .
\end{aligned}
$$


Since for each $u$ we have $P\left(X_{n}(t) \leqslant y \mid X_{n}(s)=u\right) \rightarrow P\left(Z_{u}(t-s) \leqslant y\right)$ and, since the map $u \rightarrow P\left(Z_{u}(t) \leqslant x\right)$ is bounded and continuous, we obtain (ii) from the definition of weak convergence.

In order to prove (iii) for the one-dimensional distributions write, for $0<h<t$ and $x \in \mathscr{C}_{t}$,

$$
\begin{aligned}
P(\hat{Z}(t) \leqslant x) & =\int_{-\infty}^{0} P(\hat{Z}(h) \in \mathrm{d} u) P\left(Z_{u}(t-h) \leqslant x\right) \\
& \geqslant \int_{-\infty}^{-M} P(\hat{Z}(h) \in \mathrm{d} u) P\left(Z_{u}(t-h) \leqslant x\right) \\
& \geqslant P\left(Z_{-M}(t-h) \leqslant x\right) P(\hat{Z}(h) \leqslant-M) \rightarrow P(Z(t) \leqslant x),
\end{aligned}
$$

by letting first $h \downarrow 0$ and then $M \rightarrow \infty$. On the other hand

$$
\begin{aligned}
P(\hat{Z}(t) \leqslant x) & =\int_{-\infty}^{0} P(\hat{Z}(h) \in \mathrm{d} u) P\left(Z_{u}(t-h) \leqslant x\right) \\
& \leqslant P(Z(t-h) \leqslant x) \rightarrow P(Z(t) \leqslant x) .
\end{aligned}
$$

Hence the distribution of $\hat{Z}(t)$ coincides with that of $Z(t)$. Statement (iii) for $t w o$-dimensional distributions and also for arbitrary finite-dimensional distributions is now an easy consequence of (ii) and the equality of the one-dimensional distributions at each positive time $t$.

Remark 2. The above proof is an adaption of the proof of Theorem 1 of Donnelly (1991). One of the differences is that in the present paper the state space of the Markov process is a subset of $\mathbf{R}$, whereas Donnelly treats countable state spaces; also the way we prove tightness on $D(0, \infty)$ differs from Donnolly's approach.

Corollary 1. For $F \in \mathscr{D}\left(\Psi_{\gamma}\right)$ and $\left(X_{n}\right)$, with $x_{0}<r /(1-\alpha)$, the sequence defined in (1), we have

$$
a_{n}^{-1}\left(X_{n}-\frac{b_{n}}{1-\alpha}\right) \stackrel{d}{\rightarrow} X
$$

where the limit $X$ has density $h_{\alpha}$ on $(-\infty, 0)$, given by the unique density solution of the functional equation

$$
h_{\alpha}(x)=\frac{\gamma}{|x|} \int_{x / \alpha}^{x}|x-\alpha u|^{\gamma} h_{\alpha}(u) \mathrm{d} u, \quad x<0 .
$$

Proof. For $x<0$, an elementary argument using the definition of $Z(\cdot)$ gives, for $h \rightarrow 0$,

$$
P(Z(t+h)>x)-P(Z(t)>x)=h \int_{x / \alpha}^{x}|x-\alpha u|^{\gamma} P(Z(t) \in \mathrm{d} u)+\mathrm{o}(h) .
$$


This equation can be rewritten, using the self-similarity of $Z(\cdot)$,

$$
P\left(Z(1)>x(t+h)^{1 / \gamma}\right)-P\left(Z(1)>x t^{1 / \gamma}\right)=h \int_{x / \alpha}^{x}|x-\alpha u|^{\gamma} P\left(Z(1) \in t^{1 / \gamma} \mathrm{d} u\right)+\mathrm{o}(h) .
$$

The functional equation (9) now follows by standard arguments and by using the equality $X \stackrel{d}{=} Z(1)$. That (9) has a unique density solution can be seen by calculating the moments

$$
\mu_{k}:=\int_{-\infty}^{0}|x|^{k \gamma} h_{\alpha}(x) \mathrm{d} x, \quad k=0,1, \ldots .
$$

It follows from (9) that

$$
\mu_{k}=\mu_{k+1} \int_{\alpha}^{1} \gamma y^{k \gamma-1}(y-\alpha)^{\gamma} \mathrm{d} y,
$$

and hence by a theorem of Carleman (cf. Feller 1971, p. 227), the moments $\mu_{0}=1, \mu_{1}, \ldots$ uniquely determine the density $h_{\alpha}$.

\section{The convergence result for type III distributions}

In this section we treat the case where $F \in \mathscr{D}(\Lambda)$. In order to define the limit process of $X_{n}(\cdot)$ for this case let $N$ be the Poisson process on $(0, \infty) \times \mathbf{R}$ with intensity measure $\mathrm{d} t \times \mathrm{d} \mu$, where $\mu(x, \infty)=\mathrm{e}^{-x}, x \in \mathbf{R}$. The point process $N_{x}$ is the restriction of $N$ to $(0, \infty) \times(x, \infty)$. On the points $\left.\left(t_{1}, j_{1}\right), t_{2}, j_{2}\right), \ldots$, of $N_{x}$, we define $Z_{x}(\cdot)$ by (4). Further we denote by $Z(\cdot)$ the almost sure limit of $Z_{x}(\cdot)$, as $x \rightarrow-\infty$. Along the lines of Section 2 we have the following.

Theorem 2. Let $F \in \mathscr{D}(\Lambda)$ and $x_{0}<r /(1-\alpha)$. On $D(0, \infty)$ we have

$$
X_{n}(\cdot) \stackrel{d}{\rightarrow} Z(\cdot) .
$$

Corollary 2. For $F \in \mathscr{D}(\Lambda)$ and $\left(X_{n}\right)$, with $x_{0}<r /(1-\alpha)$, the sequence defined in (1), we have

$$
a_{n}^{-1}\left(X_{n}-\frac{b_{n}}{1-\alpha}\right) \stackrel{d}{\rightarrow} X,
$$

where the limit $X$ has density $h_{\alpha}$ on $\mathbf{R}$ given by

$$
h_{\alpha}(x):=(1-\alpha)\left\{\Gamma\left((1-\alpha)^{-1}\right)\right\}^{-1} \exp \left\{-x-\mathrm{e}^{-x(1-\alpha)}\right\}, x \in \mathbf{R},
$$

and where $\Gamma(t):=\int_{0}^{\infty} x^{t-1} \mathrm{e}^{-x} \mathrm{~d} x, t>0$. 
Proof. For $x \in \mathbf{R}$ and $h \rightarrow 0$,

$$
P(Z(t+h)>x)-P(Z(t)>x)=h \int_{-\infty}^{x} \exp \{-(x-\alpha u)\} P(Z(t) \in \mathrm{d} u)+\mathrm{o}(h) .
$$

From (11) the density of $X \stackrel{d}{=} Z(1)$ can be obtained, using the self-similarity of $\exp \{-Z(t)\}$.

Remark 3. Note that the density in (10) has the form

$$
h_{\alpha}(x) \mathrm{d} x=c \exp (-\alpha x) \mathrm{d} \Lambda\{x(1-\alpha)\}, \quad \alpha \in[0,1) .
$$

However, for $\alpha \neq 0$ this density is not of the Gumbel type, i.e., there are no constants $a$ and $b$ such that

$$
h_{\alpha}(x) \mathrm{d} x=\mathrm{d} \Lambda(\alpha x+b) .
$$

\section{Tightness of sequences}

In this section we prove tightness for the sequence

$$
a_{n}^{-1}\left(X_{n}-\frac{b_{n}}{1-\alpha}\right),
$$

with $\left(X_{n}\right)$ the sequence given by (1).

Theorem 3. For $F \in \mathscr{D}\left(\Psi_{\gamma}\right)$ and $x_{0}<r /(1-\alpha)$, there exist norming constants $a_{n}>0$ and $b_{n} \in \mathbf{R}$ such that the sequence $\left\{X_{n}-b_{n} /(1-\alpha)\right\} / a_{n}$ is tight on $(-\infty, 0)$. A possible choice of $\left(a_{n}\right)$ and $\left(b_{n}\right)$ is

$$
b_{n} \equiv r, a_{n}:=r-\inf \left\{x: 1-F(x) \leqslant n^{-1}\right\} .
$$

Proof. Note by induction that $X_{n} \leqslant x_{0} \vee M_{n} /(1-\alpha)$, where $M_{n}=Y_{1} \vee Y_{2} \cdots \vee Y_{n}$, however, it is not possible to obtain a lower bound for $X_{n}$ in terms of $M_{n}$. From the well known extreme value limit for $\left(M_{n}-b_{n}\right) / a_{n}$ we obtain 0 as a distributional upper bound for $\left\{X_{n}-b_{n} /(1-\alpha)\right\} / a_{n}$.

Choose a sequence $\theta_{n}$ of positive real numbers with $a_{n} / \theta_{n} \rightarrow 1$, and satisfying

$$
\lim _{n \rightarrow \infty} n\left(1-\frac{\theta_{n+1}}{\theta_{n}}\right)=\gamma^{-1} .
$$

This is possible since $a_{n}=a(n)$, where

$$
a(y):=r-\inf \left\{x: 1-F(x) \leqslant y^{-1}\right\}, \quad y \geqslant 1,
$$

and $a$ is regularly varying; for details see Galambos and Seneta (1973) and de Bruijn (1959). Our goal is to prove that there exists a constant $A_{0}>0$ and an integer $n_{0}$ such that

$$
\mathrm{E} \frac{X_{n}-r /(1-\alpha)}{\theta_{n}} \geqslant-A_{0}, \quad n \geqslant n_{0} .
$$


This inequality, together with the upper bound $X_{n} \leqslant x_{0} \vee M_{n} /(1-\alpha)$, implies tightness of $\left\{X_{n}-b_{n} /(1-\alpha)\right\} / \theta_{n}$ and hence of $\left\{X_{n}-b_{n} /(1-\alpha)\right\} / a_{n}$, since $a_{n} / \theta_{n} \rightarrow 1$. So all we need to prove is inequality (13).

Choose $A_{1}>0$ with $A_{1}^{\gamma}=\frac{3}{2}\left[\{(\gamma+1) / \gamma\}(1-\alpha)^{-1-\gamma}\right]$, and put $\eta=(4 \gamma)^{-1}$. Since $n\left\{1-F\left(r-\theta_{n} z\right)\right\}$ converges uniformly to $z^{\gamma}$ on compacta, we can find $n_{1}$ such that, for $n \geqslant n_{1}$,

$$
\begin{aligned}
\frac{1}{A_{1}} \int_{0}^{A_{1}(1-\alpha)} n\left\{1-F\left(r-\theta_{n} z\right)\right\} \mathrm{d} z & \geqslant \frac{1}{A_{1}} \int_{0}^{A_{1}(1-\alpha)}\left(z^{\gamma}-\eta\right) \mathrm{d} z \\
& =\frac{1}{\gamma+1} A_{1}^{\gamma}(1-\alpha)^{\gamma+1}-(1-\alpha) \eta \\
& \geqslant \frac{3}{2 \gamma}-\eta \\
& =\frac{5}{4 \gamma} .
\end{aligned}
$$

According to (12) we can find $n_{2}$ such that, for $n \geqslant n_{2}$,

$$
n\left(1-\frac{\theta_{n+1}}{\theta_{n}}\right) \leqslant \gamma^{-1}+\eta=\frac{5}{4 \gamma} .
$$

Hence for $n \geqslant n_{0}=n_{1} \vee n_{2}$,

$$
\frac{1}{A_{1}} \int_{0}^{A_{1}(1-\alpha)} n\left\{1-F\left(r-\theta_{n} z\right)\right\} \mathrm{d} z \geqslant \frac{5}{4 \gamma} \geqslant n\left(1-\frac{\theta_{n+1}}{\theta_{n}}\right) .
$$

We are now ready to show (13). Note that

$$
\mathrm{E}\left(X_{n+1} \mid X_{n}\right)=X_{n}+\int_{(1-\alpha) X_{n}}^{r}\{1-F(y)\} \mathrm{d} y ;
$$

so by taking double expectations and using the Jensen inequality

$$
\mathrm{E} X_{n+1}=\mathrm{E} g\left(X_{n}\right) \geqslant g\left(\mathrm{E} X_{n}\right),
$$

where $\quad g(u):=u+\int_{(1-\alpha) u}^{r}\{1-F(y)\} \mathrm{d} y, \quad u<r /(1-\alpha)$. Put $\quad u_{n}:=\mathrm{E} X_{n} \quad$ and $\quad v_{n}:=$ $r /(1-\alpha)-A_{0} \theta_{n}$, where $A_{0}>A_{1}$ is taken large enough to satisfy

$$
u_{n_{0}} \geqslant v_{n_{0}} \text {. }
$$

We shall prove by induction that

$$
u_{n} \geqslant v_{n}
$$

for all $n \geqslant n_{0}$. Assuming that (16) holds for some $n \geqslant n_{0}$ it follows from the monotonicity of $g$ on $(-\infty, r /(1-\alpha))$ and (15) that

$$
u_{n+1} \geqslant g\left(u_{n}\right) \geqslant g\left(v_{n}\right) .
$$

Hence we shall obtain $u_{n+1} \geqslant v_{n+1}$ if we show that

$$
g\left(v_{n}\right) \geqslant v_{n+1}, \quad \forall n \geqslant n_{0} .
$$


The inequality (17) is equivalent to

$$
v_{n}+\int_{(1-\alpha) v_{n}}^{r}\{1-F(y)\} \mathrm{d} y \geqslant v_{n+1}, \quad \forall n \geqslant n_{0},
$$

or, after setting $y=r-A_{0} \theta_{n} z$,

$$
\int_{0}^{1-\alpha} n\left\{1-F\left(r-A_{0} \theta_{n} z\right)\right\} \mathrm{d} z \geqslant n\left(1-\frac{\theta_{n+1}}{\theta_{n}}\right), \quad \forall n \geqslant n_{0} .
$$

Inequality (18), and hence (17), follows from

$$
\begin{aligned}
\int_{0}^{1-\alpha} n\left\{1-F\left(r-A_{0} \theta_{n} z\right)\right\} \mathrm{d} z & \geqslant \int_{0}^{1-\alpha} n\left\{1-F\left(r-A_{1} \theta_{n} z\right)\right\} \mathrm{d} z \\
& =\frac{1}{A_{1}} \int_{0}^{(1-\alpha) A_{1}} n\left\{1-F\left(r-\theta_{n} z\right)\right\} \mathrm{d} z \\
& \geqslant n\left(1-\frac{\theta_{n+1}}{\theta_{n}}\right),
\end{aligned}
$$

for all $n \geqslant n_{0}$, according to (14).

The proof of tightness of the sequence $a_{n}^{-1}\left\{X_{n}-b_{n} /(1-\alpha)\right\}$, in case $F \in D(\Lambda)$, can be given in a similar way; therefore we omit this proof.

Theorem 4. For $F \in \mathscr{D}(\Lambda)$ and $x_{0}<r /(1-\alpha), \quad\left(a_{n}\right)$ and $\left(b_{n}\right)$ such that $F^{n}\left(a_{n} x+\right.$ $\left.b_{n}\right) \rightarrow \Lambda(x)$ we have that $\left\{X_{n}-b_{n} /(1-\alpha)\right\} / a_{n}$ is tight on $\mathbf{R}$.

\section{Concluding remarks}

(i) Together with the paper of Greenwood and Hooghiemstra (1991) this paper gives sufficient conditions on $F$ to ensure that $\left\{X_{n}-b_{n} /(1-\alpha)\right\} / a_{n}$ has a distributional limit. It is known that for $\alpha=0$ these conditions are also necessary. Whether this is also the case for $0<\alpha<1$ we do not know.

(ii) The recursion (1) can be written as

$$
X_{n}=X_{n-1}+\left[Y_{n}-(1-\alpha) X_{n}\right]^{+} .
$$

A description of what kind of results can be expected if we let $\alpha$ depend on $n$ such that $\alpha_{n} \rightarrow 1$ is given in the work of den Hollander et al. (1991). 


\section{Acknowledgements}

We would like to thank Tom Liggett for the original proof of Lemma 2. Discussions with Serguei Foss, Michael Keane and Laurens de Haan have been extremely valuable to us.

\section{References}

de Bruijn, N.G. (1959) Pairs of slowly oscillating functions occurring in asymptotic problems concerning Laplace transforms. Nieuw Arch. Wisk., 7, 20-26.

Donnelly, P. (1991) Weak convergence to a Markov chain with an entrance boundary; ancestral processes in population genetics. Ann. Probab., 19, 1102-1117.

Feller, W. (1971) An Introduction to Probability Theory and its Applications, Vol. II. New York: Wiley.

Galambos, J. and Seneta, E. (1973) Regularly varying sequences. Proc. Amer. Math. Soc., 41, 110116.

Greenwood, P.E. and Hooghiemstra, G. (1991) On the domain of an operator between supremum and sum. Probab. Theory Related Fields, 89, 201-210.

Haslett, J. (1980) Problems in the stochastic storage of solar thermal energy. In O.L.R. Jacobs, M.H.A. Davies, M.A.H. Dempster, C.J. Harris and P.C. Parks (eds) Analysis and Optimization of Stochastic Systems, pp. 553-562. London: Academic Press.

den Hollander, F., Hooghiemstra, G., Keane, M. and Resing, J. (1991) Strong law and central limit theorem for a process between maxima and sums. Probab. Theory Related Fields, 90, 37-55.

Received May 1996 and revised October 1996 